\title{
Adding Two Dimensions to Heart Rate Variability Research
}

\author{
Joachim A Behar $^{1 *}$, Ori Shemla ${ }^{1 *}$, Ido Weiser-Bitoun ${ }^{1}$, Aviv A Rosenberg ${ }^{2}$ and Yael Yaniv ${ }^{1}$ \\ ${ }^{1}$ Faculty of Biomedical Engineering, Technion, Israel Institute of Technology, Haifa, Israel \\ ${ }^{2}$ Department of Computer Science, Israel Institute of Technology, Haifa, Israel \\ "Equal contribution
}

\begin{abstract}
Introduction: Heart rate variability (HRV) analysis tools have been mainly available for analysis of human electrocardiographic derived heart rate. We explore extending HRV analysis to two additional dimensions: (1) analysis across multiple mammalian species and (2) analysis across different levels of integration for example sinoatrial tissue.

Methods: We analyzed the beating rate variability (BRV) across the two additional dimensions using the PhysioZoo computer program that we recently introduced. We used published databases of electrocardiograms from four mammal types: human $(n=18)$, dog $(n=17)$, rabbit $(n=4)$ and mouse $(n=8)$. We computed the BRV measures for each. We also show how the PhysioZoo program can be used for the analysis of sinoatrial node tissue BRV.

Results: The study of typical mammalian heart and respiration rates (obtained from the dominant high frequency peak) revealed a linear relationship between these two quantities. Analysis of the rabbit sinoatrial node tissue BRV showed that it had reduced overall variability when compared to in vivo heart BRV.
\end{abstract}

\section{Introduction}

Heart rate variability (HRV) analysis is a common tool in cardiovascular physiology research because it is a noninvasive method for assessing heart function. More generally speaking, the beating rate variability (BRV) of the heart in vivo, sinoatrial node or single pacemaker cells can be studied to investigate cardiovascular function [1]. In this paper we use the term HRV to describe beat-to-beat interval variations from in-vivo ECG data and BRV as a more generic term to denote beat-to-beat interval variations from any electrophysiological signal (including signals from sinoatrial node cells, sinoatrial tissue, and in vivo hearts).

Recently we introduced the PhysioZoo platform [2], an open-source program dedicated to the analysis of the beating rate variability (BRV) in mammalian electrophysiological recordings (physiozoo.com). The platform includes open-source software (code and user interface) for the analysis of the BRV in mammals and a set of annotated databases of electrophysiological signals. The software includes all the standard HRV measures, adapted for use with different mammals (humans, rabbits, dogs, and mice). Adapting these measures to other mammals is also possible in this framework. The PhysioZoo project aims to standardize BRV analysis from mammalian electrophysiological data, enable its reproducibility, and enable its use across multiple mammalian species and different levels of integration (i.e. sinoatrial cell, tissue and in vivo electrophysiological signals). In its current version the software readily implements all the steps of BRV analysis (peak detection, prefiltering and HRV measures) using electrocardiogram (ECG) recordings from different mammals.

In this paper we illustrate how to use PhysioZoo to analyze BRV across species or across levels of integration and discuss the physiological insights that can be obtained. For that purpose, we explore the relationship between the typical heart rate $\left(\mathrm{HR}_{\mathrm{m}}\right)$ and breathing rate $\left(\mathrm{BR}_{\mathrm{m}}\right)$ across different mammalian species and the difference in BRV as obtained from rabbit ECG vs. spontaneously beating rabbit sinoatrial node tissue electrogram.

\section{Methods}

Animal data from multiple studies [2]-[6] were collected for analysis. All animal studies were performed in accordance with the Guide for the Care and Use of Laboratory Animals published by the National Institutes of Health (NIH Publication no.85-23, revised1996).

We analyzed the beating rate variability (BRV) using the PhysioZoo program. We used the published PhysioZoo databases (Table 1) of electrocardiograms from four mammal types: humans $(\mathrm{n}=18), \operatorname{dogs}(\mathrm{n}=17)$, rabbits $(n=4)$, and mice $(n=8)$ [2]. These databases have reference peak annotations (i.e. manually corrected). The AVNN and high frequency (HF) peaks were computed and exported from the PhysioZoo program. We looked for allometric laws between the typical heart rate $\left(H R_{m}\right)$ and the typical body mass $\left(B M_{m}\right)$ and between the breathing rate $\left(B R_{m}\right)$ 
and $B M_{m} . B M_{m}$ values were taken from [7]. We looked for a linear relationship between the $H R_{m}$ of different mammals and their typical $B R_{m}$. The breathing rate was evaluated by detecting the dominant peak location in the HF band of the power spectrum, which is known to be characteristic of respiratory sinus arrythmia [8]. Finally, we looked at the mean normalized power spectral density (PSD) for each mammalian database included.

We illustrate how sinoatrial node tissue beating variability can be analyzed and use the rabbit electrogram sample to show the difference in overall variability (quantified using SDNN) between sinoatrial node tissue electrogram data and in-vivo electrocardiogram data.

Accurate BRV analysis requires the following steps: accurate peak detection, preprocessing to remove sudden drops and increases in the RR intervals due to ectopic beats and noise, selection of the analysis window, and computation of HRV measures (Figure 1). The algorithms are readily available in PhysioZoo for HRV analysis. Figure 2 illustrates the PhysioZoo interface for performing HRV analysis.

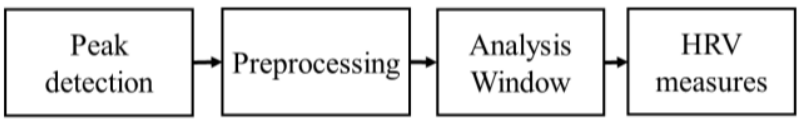

Figure 1: Performing HRV analysis in PhysioZoo.

To find the beat-to-beat intervals of the electrogram recording, the following steps were performed: recordings were prefiltered using a Notch filter at $60 \mathrm{~Hz}$ to remove power-line interference and the depolarization peaks were searched for. Depolarization peaks are sharper; thus, we used them as fiducial points to compute the beat-to-beat intervals. All detected beats were manually corrected to correct for misdetections. Then the beat-to-beat intervals were processed using PhysioZoo.

Table 1: PhysioZoo mammalian ECG database [2]-[6].

\begin{tabular}{lcccc}
\hline & Human & Dog & Rabbit & Mouse \\
\hline Number of records & 18 & 17 & 20 & 8 \\
Number of mammals & 18 & 17 & 4 & 8 \\
Total length (hr:min:sec) & $437: 29: 36$ & $01: 33: 55$ & $03: 31: 13$ & $03: 28: 07$ \\
Total R-peak annotations & $1,806,792$ & 10,871 & 50,452 & 109,865 \\
\hline
\end{tabular}

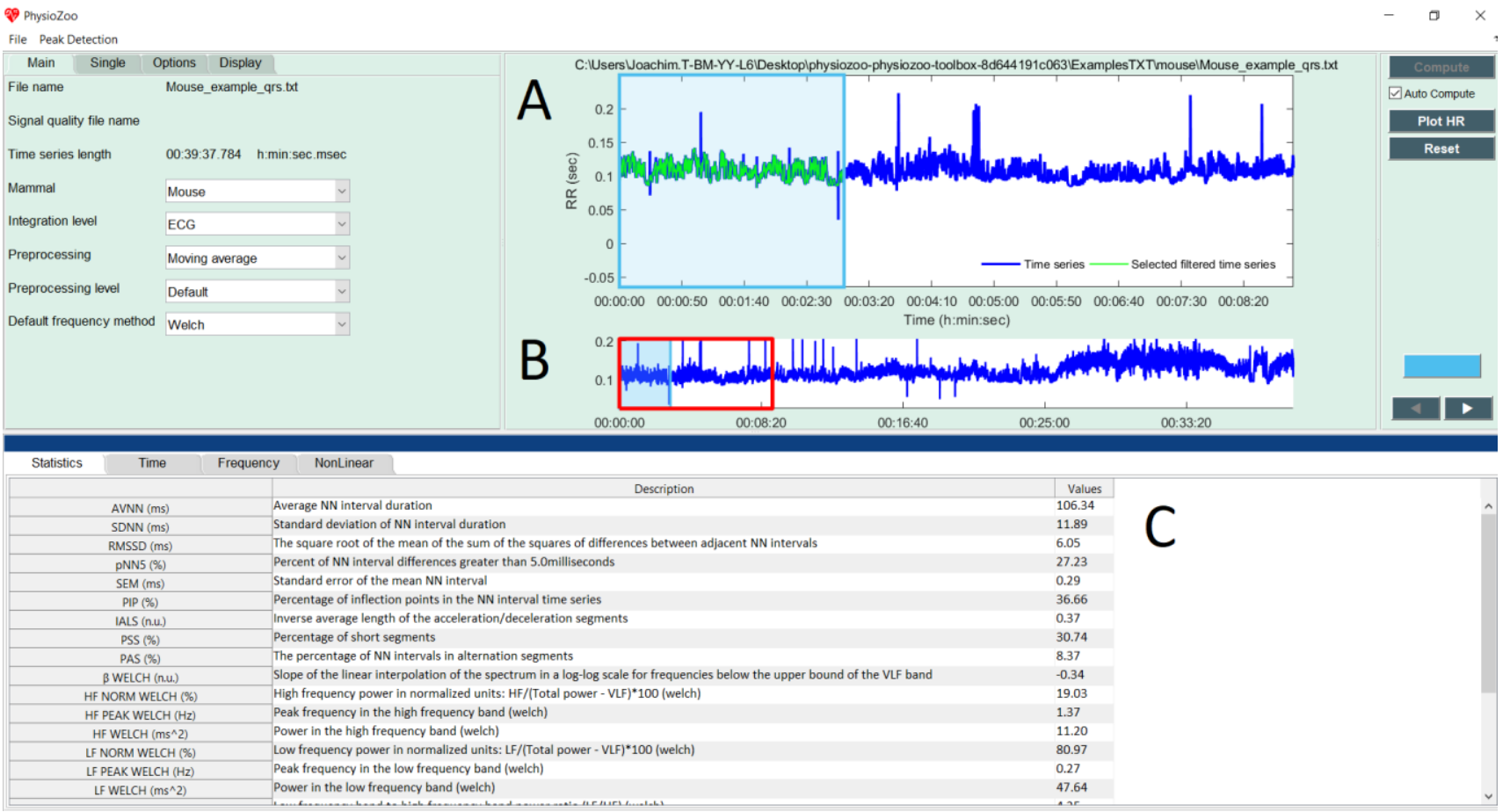

Figure 2: PhysioZoo user interface. (A) The selected analysis window (in blue) defines the time interval for which the HRV measures are computed. (B) The RR time series is plotted and two windows drawn: the red-framed window defines the part of the RR time series plotted in the larger upper figure (A), and the selected window in blue defines the part of the RR time series for which the HRV measures will be computed. The window can be modified (enlarged/decreased/moved) using the mouse. Panel (C) shows all the HRV measures that have been computed. 

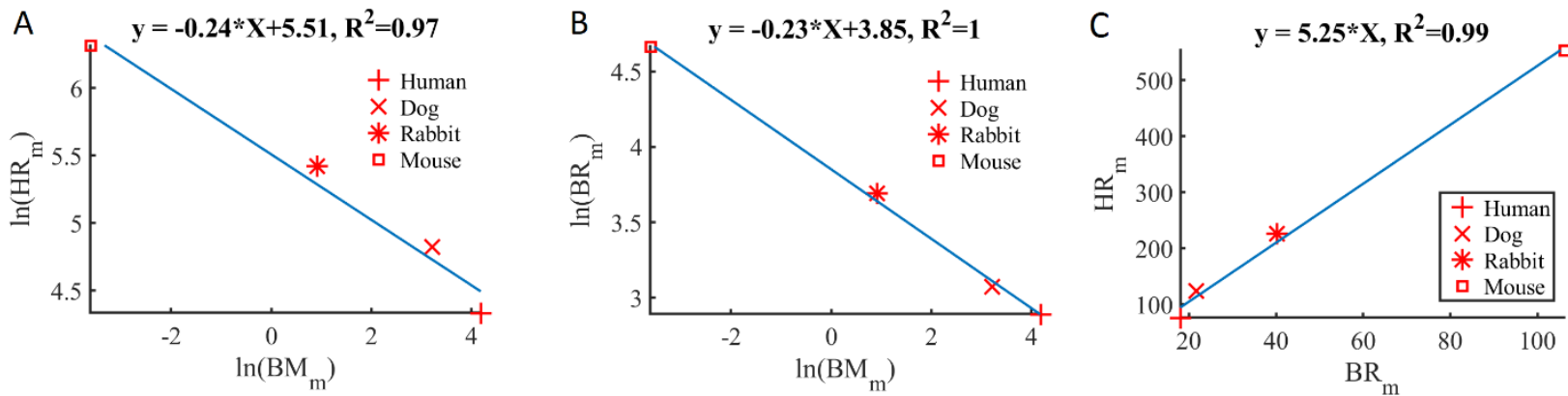

Figure 3: Power laws (A-B) and linear relationship (C) between: $(A)$ the typical mammalian heart rate $\left(H_{\mathrm{m}}\right)$ and body mass index $\left(\mathrm{BM}_{\mathrm{m}}\right),(\mathrm{B})$ the breathing rate $\left(\mathrm{BR}_{\mathrm{m}}\right)$ and $\mathrm{BM}_{\mathrm{m}},(\mathrm{C})$ the $\mathrm{HR}_{\mathrm{m}}$ and $\mathrm{BR}_{\mathrm{m}}$ of the four types of mammals . The typical $B R_{m}$ was taken as 60 times the typical PSD high frequency peak location. Both allometric laws (A-B) scale with a power of $\sim 1 / 4$ of $B M_{m}$ and thus highlight the linear relationship between the breathing rate and the heart rate (C). Thus, on average, one breathing cycle of any mammal contains the same number of heartbeats.

\section{Results}

Allometric laws found for the $\mathrm{HR}_{\mathrm{m}}$ and $\mathrm{BR}_{\mathrm{m}}$ (Figure $3 \mathrm{~A}, \mathrm{~B})$ scale with a power of $\sim 1 / 4$ of $\mathrm{BM}_{\mathrm{m}}$. This is in accordance with the allometric laws found for $\mathrm{HR}_{\mathrm{m}}$ and for $\mathrm{BR}_{\mathrm{m}}$ by others [9], [10]. We found a linear relationship between $H R_{m}$ and $B R_{m}: H R_{m}=5.25 * B R_{m}$ with $R^{2}=$ 0.99 (Figure 3C).

We looked at the mean normalized power spectral density for each mammalian database. Interestingly, our results show that the mouse could serve as a better mammalian model than the dog or rabbit for studying the effects of drugs, mutations, or cardiac diseases on vagal activity as reflected in the HF band. This is because respiratory sinus arrhythmia activity is higher in smaller mammals, and thus the high frequency peak shifts to the right as the mammalian size gets smaller (Figure 4), leading to less overlap between the physiological processes echoed in the low frequency and the high frequency bands. However, this effect is moderated by the relatively lower energy contained in the HF band for the mouse versus bigger mammals.

Finally, we illustrate how PhysioZoo can be used to analyze BRV from electrogram data (Figure 5 and Figure 6). Figure 6 shows the beat-to-beat interval variation against time from the electrogram recording when no preprocessing is applied and taking the repolarization peak as the fiducial point to be detected versus preprocessing the data with a Notch filter and taking the depolarization peak as the fiducial point. This beat detection algorithm for electrogram data will be further developed and implemented in PhysioZoo in future work. Analysis of the sample rabbit sinoatrial node tissue BRV showed that it had reduced overall variability $(\mathrm{SDNN}=5.3 \mathrm{~ms})$ in comparison to the whole innervated heart HRV (SDNN $=9.5 \mathrm{~ms}$ ).

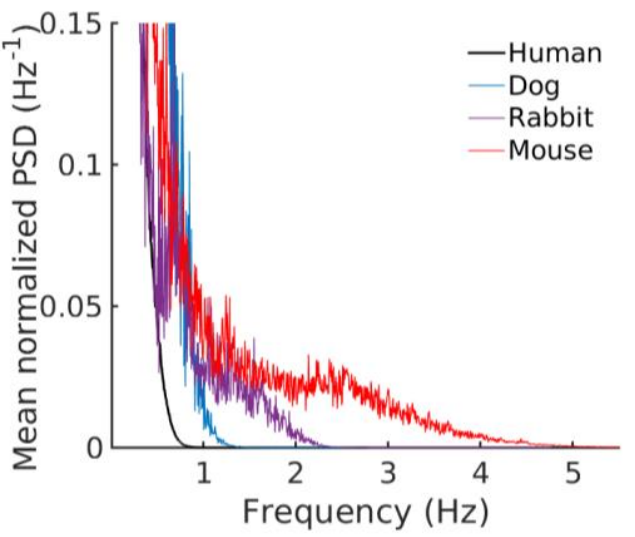

Figure 4: Mean normalized power spectral density for each mammalian database from Table 1 . Note the shift of the high frequency content to the right as the mammalian size gets smaller. Adapted from [6].

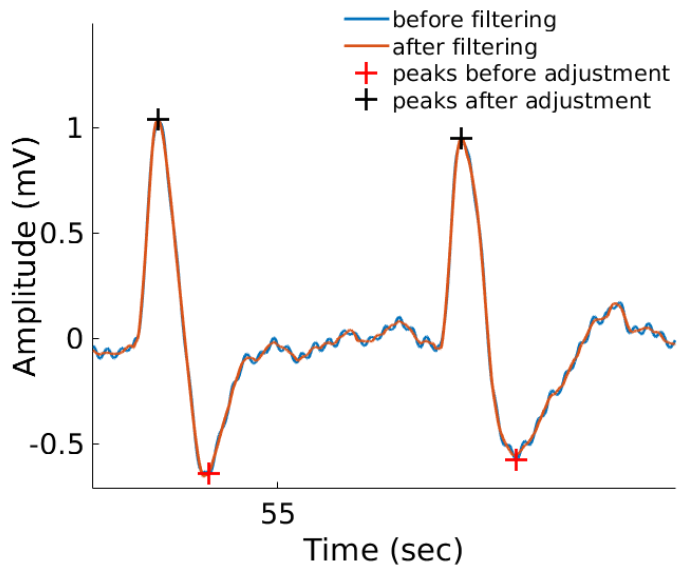

Figure 5: Beat-to-beat interval variation when detecting the depolarization peak after preprocessing with a Notch filter in an electrogram signal ('peaks after adjustment') versus detecting the repolarization peak and without preprocessing ('peaks before adjustment'). 


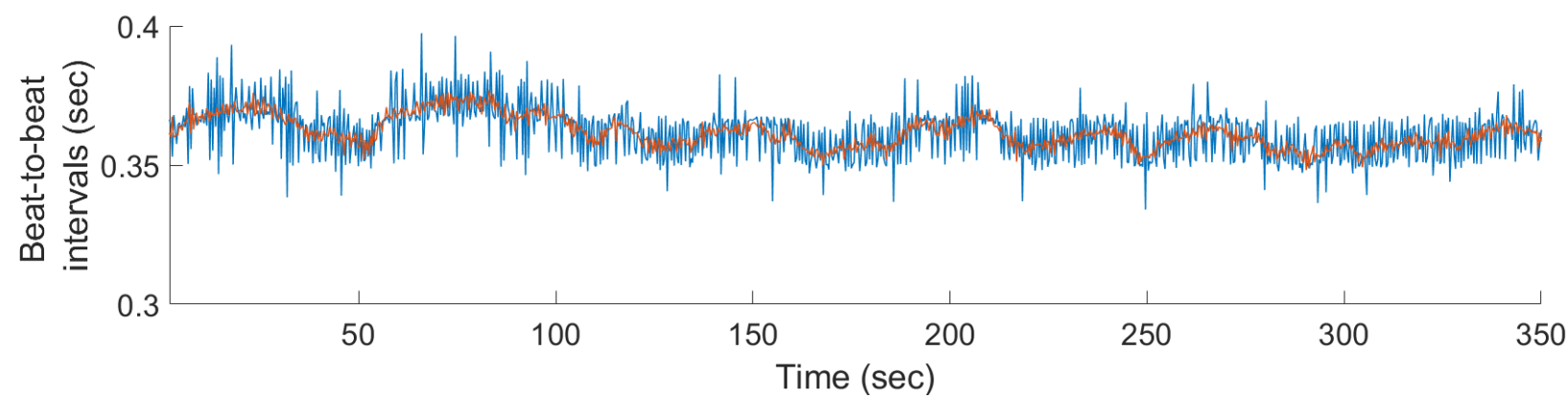

Figure 6: Beating rate variability analysis of an electrogram sample: example of applying a Notch filter (60 Hz) and looking for the depolarization peak fiducial (orange) versus detecting the repolarization fiducial and not applying a Notch filter (blue). This example highlights the importance of the preprocessing step and what fiducial point to look for in order to guarantee accurate BRV analysis. For example, $\mathrm{SDNN}=9.02 \mathrm{~ms}$ for the blue time series versus $5.3 \mathrm{~ms}$ for the orange one.

\section{Discussion and conclusion}

Our first main conclusion is that there is a linear relationship between $\mathrm{BR}_{\mathrm{m}}$ and $\mathrm{HR}_{\mathrm{m}}$. This linear relationship implies that the number of beats in one breathing cycle is the same for all mammals. It was obtained by the study of HRV across multiple species. The second main conclusion is that the separation between the high frequency band and low frequency bands in PSD analysis is better for the mouse versus the other mammals studied. This suggests that the mouse can serve as an important animal model when investigating vagal effects. Finally, we showed that the BRV was reduced in isolated sinoatrial nodes versus the in-vivo heart. We observed that the analysis of electrophysiological signals other than ECG requires adaptation of preprocessing strategies in order to obtain a meaningful beat-to-beat time series (Figure 6) and thus accurate BRV analysis. This will be further developed and implemented in PhysioZoo.

\section{Acknowledgements}

This work was supported by the Center for Absorption in Science, Ministry of Immigrant Absorption, State of Israel (JB), the Technion E.V.P.R. Star Fund (YY), Ilene and Steve Berger Fund (YY), and NSFC-ISF Joint Research Program, No. 398/14 (YY).

\section{References}

[1] Y. Yaniv et al., "Synchronization of sinoatrial node pacemaker cell clocks and its autonomic modulation impart complexity to heart beating intervals," Heart. Rhythm, vol. 11, no. 7, pp. 1210-19, 2014.

[2] J. Behar et al., "PhysioZoo: a novel open access software and databases for heart rate variability analysis in mammals," Accept. Publ. Front. Physiol.

[3] Y. Yaniv et al., "Deterioration of autonomic neuronal receptor signaling and mechanisms intrinsic to heart pacemaker cells contribute to age-associated alterations in heart rate variability in vivo," Aging Cell, vol. 15, no. 4, pp. 716-724, 2016.

[4] M. Brunner et al., "Mechanisms of cardiac arrhythmias and sudden death in transgenic rabbits with long QT syndrome.," J. Clini. Invest., vol. 118, no. 6, pp. 224659, Jun. 2008.

[5] G. E. Billman, H. V. Huikuri, J. Sacha, and K. Trimmel, "An introduction to heart rate variability: methodological considerations and clinical applications," Front. Physiol., vol. 6, p. 55, Feb. 2015.

[6] J. Behar et al., "A universal scaling relation for defining power spectral bands in mammalian heart rate variability analysis," Front. Physiol., vol. 9, p. 1001, 2018.

[7] University of Wisconsin-Madison, "Animal Health: Normative Data," Research Animal Resources Center. University of Wisconsin-Madison, 2018. Available: https://www.rarc.wisc.edu/animal_health/normative_da ta.html.

[8] S. Akselrod, D. Gordon, F. A. Ubel, D. C. Shannon, A C. Berger, and R. J. Cohen, "Power spectrum analysis of heart rate fluctuation: a quantitative probe of beat-tobeat cardiovascular control," Science., vol. 213, no. 4504, pp. 220-222, 1981.

[9] K. Schmidt-Nielsen, Scaling: why is animal size so important? Cambridge University Press, 1984.

[10] W. R. Stahl, "Scaling of respiratory variables in mammals," J. Appl. Physiol., vol. 22, no. 3, pp. 453460, Mar. 1967.

Joachim A. Behar, PhD

Post-doctoral fellow, Technion-IIT, Haifa, Israel

The Technion Bioelectric and Bio-energetic Systems Laboratory jbehar@technion.ac.il 